\title{
Funcionamiento familiar relacionado con el riesgo suicida en adolescentes de una secundaria en Aguascalientes
}

Quezada-Martín Nancy Guadalupe*, Hernández-Sandoval Gladys Berenice*, Hernández-Jacinto Blanca Denisse*, Martínez-Díaz Laura Elizabeth*, Mejía-Guerrero Lourdes**, Guerrero-Mojica Nery**

\begin{tabular}{|c|c|}
\hline 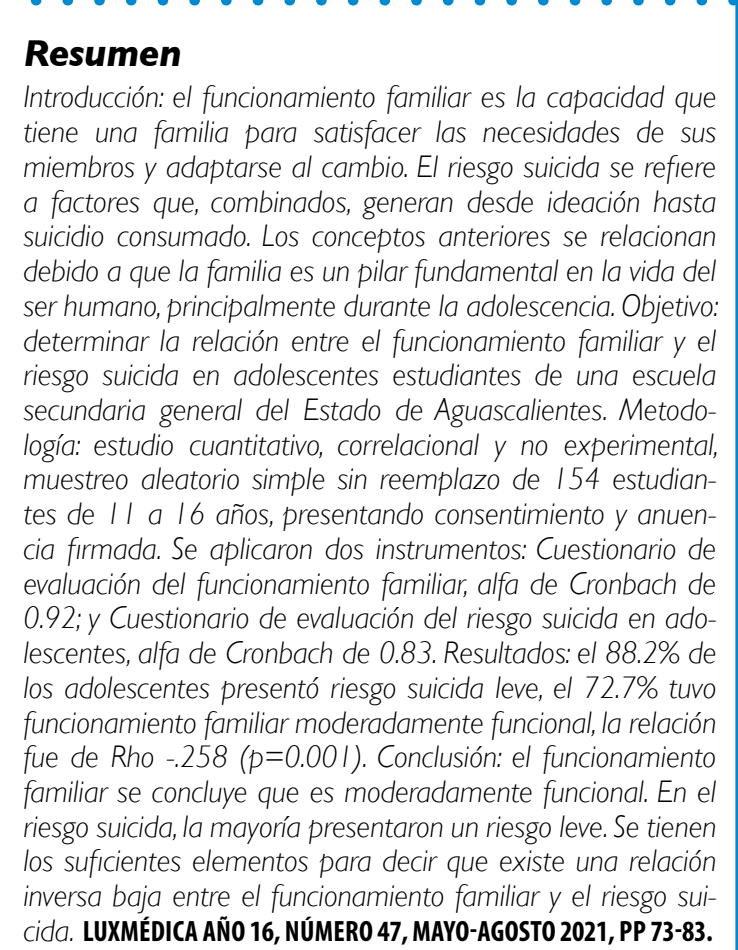 & 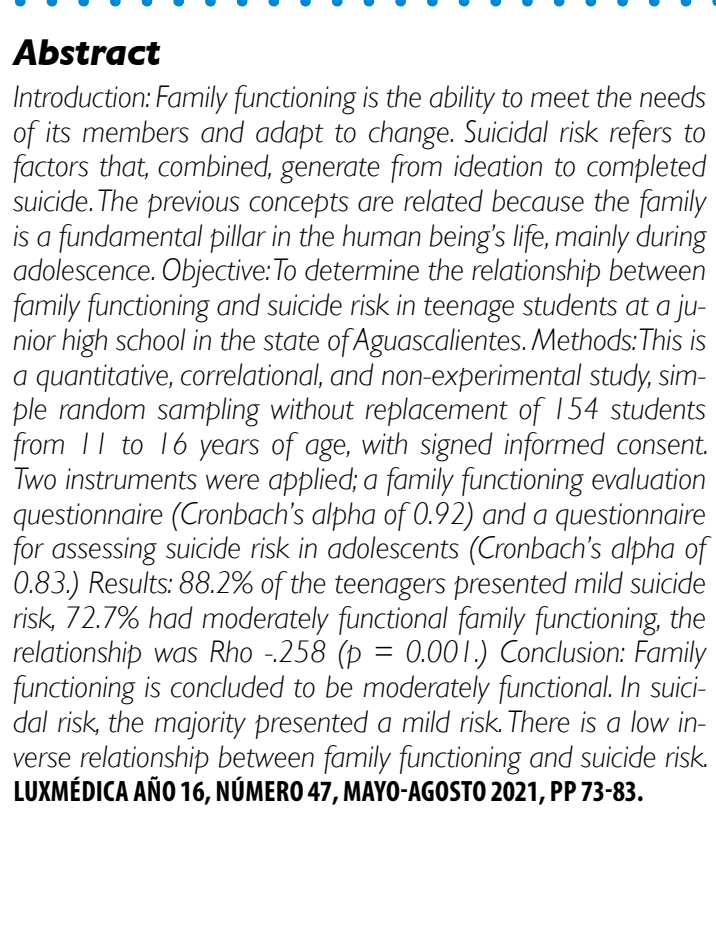 \\
\hline$\lambda$ & \\
\hline
\end{tabular}

* $\quad$ Estudiante de la Lic. en Enfermería del Centro de Ciencias de la Salud de la Universidad Autónoma de Aguascalientes. https://orcid.org/0000-00034423-8232. Correo electrónico sita_nancy2@outlook.com

* Estudiante de la Lic. en Enfermería del Centro de Ciencias de la Salud de la Universidad Autónoma de Aguascalientes. https://orcid.org/0000-00017620-1333. Correo electrónico gbhernandez2118@gmail.com

Estudiante de la Lic. en Enfermería del Centro de Ciencias de la Salud de la Universidad Autónoma de Aguascalientes. https://orcid.org/0000-00027292-7388. Correo electrónico bd.hdezj20@gmail.com

* $\quad$ Estudiante de la Lic. en Enfermería del Centro de Ciencias de la Salud de la Universidad Autónoma de Aguascalientes. https://orcid.org/0000-00020219-0827. Correo electrónicolaurinchiz04@gm

** $\quad$ Profesora investigadora del Departamento de Enfermería del Centro de Ciencias de la Salud de la Universidad Autónoma de Aguascalientes. https:// orcid.org/0000-0003-0580-4086. Correo electrónicog.n.ery@hotmail.com

** $\quad$ Profesora investigadora del Departamento de Enfermería del Centro de Ciencias de la Salud de la Universidad Autónoma de Aguascalientes. https:// orcid.org/0000-0003-1039-2223. Correo electrónico lulamejia@gmail.com

Fecha de recibido: 15 de octubre 2020

Fecha de aceptación: 20 de febrero 2021

Correspondencia: MCE Nery Guerrero Mojica. Departamento de Enfermería, Edificio 105, Centro de Ciencias de la Salud. Universidad Autónoma de Aguascalientes. Av. Universidad número 940. Ciudad universitaria. CP: 20131 Aguascalientes Ags. México. Teléfono: 449-910-84-37 Correo electrónico: g.n.ery@hotmail.com 


\section{Introducción}

El suicidio es un fenómeno tan antiguo como la existencia misma de la humanidad. En el año 2015, a nivel mundial, el suicidio ocupaba la tercera causa de muerte en adolescentes, con 67000 casos. En México, para 2018, ocupó el cuarto lugar de las principales causas de mortalidad en el grupo de niños y adolescentes de 10 a 17 años, con una tasa de mortalidad de 3.6 fallecimientos por cada 100 mil niños y adolescentes. ${ }^{1-2}$

Los estados con mayor incidencia de suicidios en México son: Chihuahua con el primer lugar, seguido de Aguascalientes y en tercer lugar, Campeche. El estudio presentado por el Instituto $\mathrm{Na}$ cional de Estadística y Geografía (INEGI) en 2015, muestra que el estado de Aguascalientes ocupa el segundo lugar a nivel nacional en suicidios de jóvenes de 15 a 29 años. ${ }^{3}$

Según la OMS, las causas más comunes de suicidio en los adolescentes son acoso por parte de iguales o bullying, maltrato físico y abuso sexual, dificultades con el grupo de iguales y pareja, sucesos vitales estresantes y antecedentes psiquiátricos y suicidio en la familia. La adolescencia es el periodo de crecimiento y desarrollo humano que se produce después de la niñez y antes de la edad adulta, entre los 10-19 años. ${ }^{4-5}$ Se considera la etapa de transición más importante en la vida del ser humano, se caracteriza por un ritmo acelerado de crecimiento y de cambios. ${ }^{5}$

El funcionamiento familiar es la capacidad que tiene la familia para satisfacer las necesidades de sus miembros y adaptarse a las situaciones de cambio. La familia se considera funcional cuando es capaz de propiciar la solución a los problemas, de modo que éstos no lleguen a afectar la satisfacción de las necesidades de sus miembros. ${ }^{6}$

El riesgo suicida está constituido por factores que se combinan entre sí, que puede atravesar por fases de ideación, planeación, intento y suicidio consumado. ${ }^{7-8}$

En la investigación Relación entre ideación suicida, depresión y funcionamiento familiar en adolescentes realizada por Luz de Lourdes Eguiluz Romo, los resultados demostraron que, a mayor ideación suicida, menor es la probabilidad de un involucramiento afectivo funcional y, a mayor ideación suicida, menores serán los patrones de comunicación funcionales. ${ }^{8}$

El suicidio es un problema relevante en la época actual, por lo que se ha denominado como un problema de salud pública, en esta investigación se hace énfasis al funcionamiento familiar ya que la familia es el soporte y un factor importante para evitar que este problema se desarrolle. ${ }^{9}$

Se decide abordar este tema de investigación ya que Aguascalientes se encuentra en segunda posición con la mayor tasa de suicidios a nivel nacional ${ }^{10}$ y se considera que con esta investigación enfocada en adolescentes se puede abonar a la detección del riesgo de manera oportuna, además de poder detectar problemas en el funcionamiento familiar que lo desencadenan. 


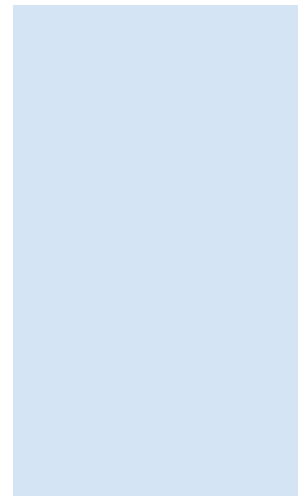

En cuanto a la hipótesis de investigación se tomará en cuenta que el funcionamiento familiar tiene relación con el riesgo suicida en adolescentes de una secundaria en Aguascalientes.

La pregunta de investigación es: ¿Existe relación entre el funcionamiento familiar con el riesgo suicida en adolescentes de una secundaria en Aguascalientes?

El objetivo que se pretendía logar era: determinar la relación entre el funcionamiento familiar con el riesgo suicida en adolescentes estudiantes de una escuela secundaria general del estado de Aguascalientes, que presentan diferentes conductas suicidas como consecuencia de diversos factores influyentes.

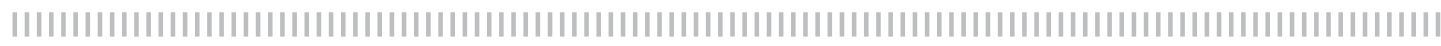

\section{Material y métodos}

Estudio cuantitativo, correlacional, transversal y prospectivo. El universo lo constituyeron 370 adolescentes de una secundaria general de Aguascalientes, de la cual se extrajo una muestra conformada por 154 adolescentes. Fueron seleccionados a través de una secuencia de números aleatorios sistemáticos, con un muestreo aleatorio simple sin reemplazo. Los criterios de inclusión que ayudaron a conformar la muestra fueron los siguientes: adolescentes de ambos sexos con la edad de 11 a 16 años cumplidos, que fueran estudiantes de una secundaria general de Ags, que desearan participar en la investigación y que se les hubiera entregado el consentimiento informado y la anuencia. Se consideró como único criterio de eliminación a aquellos que no desearan participar, aunque se les hubiera entregado el consentimiento informado y la anuencia.

El primer instrumento que se utilizó fue el Cuestionario de Evaluación del Funcionamiento Familiar ${ }^{6}$ con un alfa de Cronbach de 0.92, de escala tipo likert con cuarenta reactivos, cada uno con cinco opciones de respuesta con un rango de 1 a 5 . Incluye seis factores 1) involucramiento familiar, 2) involucramiento afectivo disfuncional 3) patrones de comunicación disfuncionales 4) patrones de comunicaciones funcionales 5) resolución de problemas 6) patrones de control de conducta. El segundo ins- trumento Detección de Riesgo Suicida en Adolescentes ${ }^{7}$ con un alfa de Cronbach de 0.83 , con veinticinco reactivos, cada uno con tres opciones de respuesta, con un rango de 0 a 2 . Se estructuró en dos partes: 22 ítem que detectaron la ideación suicida y 3 ítems de intento de suicidio. La puntuación total se obtuvo sumando el subpuntaje de ideación suicida más el puntaje de intento suicida. La suma total oscila de 0 a 50 puntos. Siendo un riesgo leve de 0 a 24 , moderado de 25 a 37 y grave de 38 a 50 puntos.

La aplicación de los instrumentos se realizó durante septiembre del 2019, después de que se entregó el consentimiento informado y la anuencia a los adolescentes. Se aplicó el instrumento a los estudiantes que lo entregaron firmado. Se dieron 10 minutos para la explicación de las instrucciones del instrumento y 20 minutos más para contestarlo. Al finalizar, se verificó que los instrumentos fueron contestados en su totalidad. Para el análisis de datos se utilizó el paquete estadístico SPSS versión 25.0, para la comprobación de la hipótesis se utilizó análisis no paramétrico: coeficiente de correlación Rho de Spearman para las variables de Funcionamiento Familiar y Riesgo Suicida. En la estadística descriptiva se utilizó distribución de frecuencias y porcentajes para las variables cualitativas y medidas de tendencia central y dispersión para variables cuantitativas. 
Se utilizó un consentimiento informado y una anuencia dirigidos hacia padres o tutores, puesto que se trabajó con menores de edad y esto representó un riesgo mínimo para su salud, todo esto conforme a la
Ley General de Salud en materia de investigación. ${ }^{11}$ Se respetaron los principios de autonomía, no maleficencia, beneficencia y justicia como se escribió en la declaración de Helsinki. ${ }^{12}$

| | | | | | | | | | | | | | | | | | | | | | | | | | | | | | | | | | | | | | | | | | | | | | | | | | | | | | | | | | | | | | | | | | | | | | | | | | | | | | | | | | | | | | | | | | | | | | | | | | | | | | | | | | | | | | | | | | | | | |

\section{Resultados}

Entre las características demográficas más relevantes de la población, se encontró que, del total de la muestra, $58.40 \%$ eran mujeres, mientras que $41.60 \%$ eran hombres. La edad promedio de los adolescentes encuestados fue de 13 años correspondiendo al $37.5 \%$ de la muestra. Con relación al tipo de familias sólo el $63.60 \%$ fue de confor- mación familiar nuclear, mientras que el $29.40 \%$ de monoparental.

En el funcionamiento familiar de los adolescentes se encontraron diferencias significativas en dos niveles de los cuatro que se tomaron en cuenta. El $72.7 \%$ presentó un nivel moderadamente funcional en comparación al $1.9 \%$ que tuvo un nivel de funcionamiento severamente disfuncional (figura 1).

\section{Gráfica 1. Evaluación del funcionamiento familiar.}

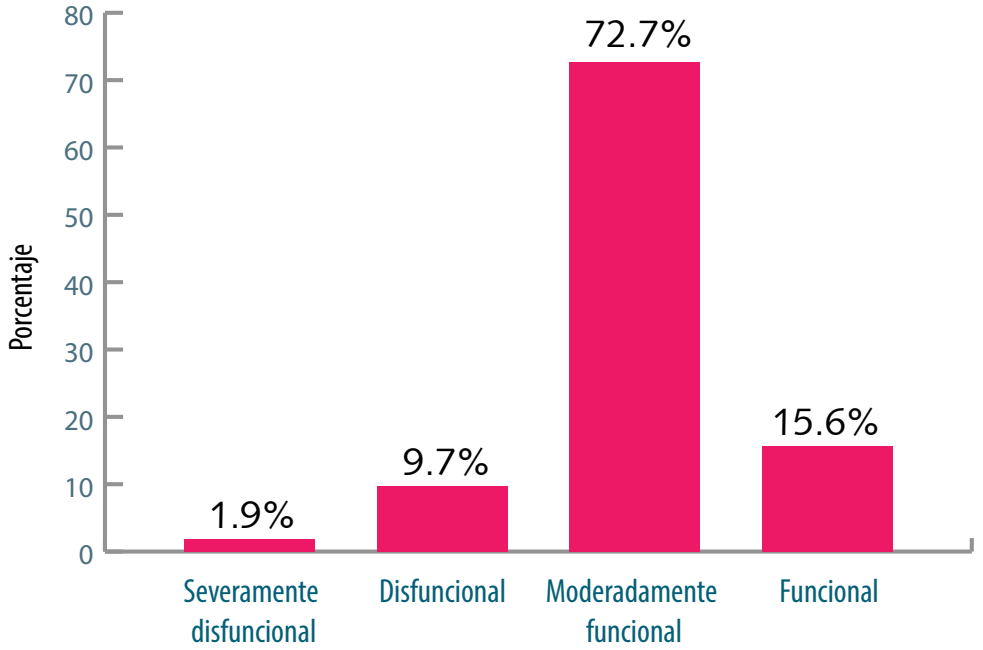

Niveles de funcionamiento familiar
Fuente: Instrumento aplicado a adolescentes de una secundaria general en Aguascalientes. Septiembre de $2019(n=154)$
Por otra parte, se puede apreciar que, del factor patrón de comunicación disfuncional, el 57.8\% mostró comunicación sí disfuncional, mientras que el otro $42.2 \%$ comunicación no disfuncional. Por lo tanto, de los factores que evalúa el instrumento Evaluación del funcionamiento familiar (involucramiento afectivo, resolución de problemas, patrones de conducta, y patrón de comunicación disfuncional) demuestra que la comunicación es la más alterada, siendo esto lo que afecta principalmente a un adecuado funcionamiento familiar (figura 2). 


\section{Gráfica 2. Patrón de comunicación disfuncional.}

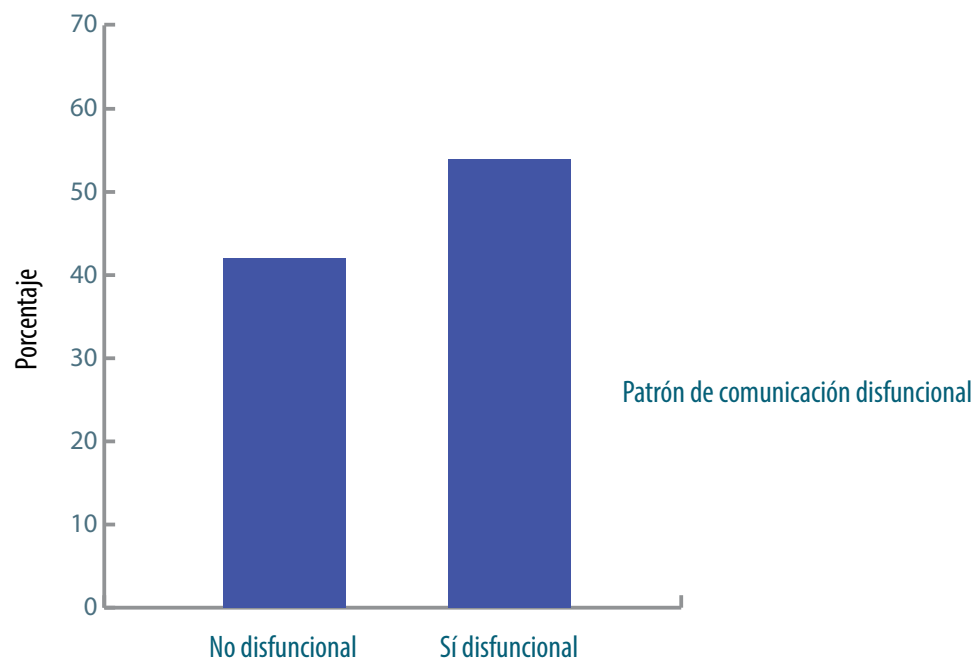

Fuente: Instrumento aplicado a adolescentes de una secundaria general en Aguascalientes. Septiembre de $2019(n=154)$

En cuanto al cuestionario para la Detecriesgo moderado y ninguno demostró tener ción de Riesgo Suicida en Adolescentes, los resultados fueron los siguientes: predominando un riesgo leve con $88.20 \%$ (136), riesgo grave. Sin embargo, existen adolescentes que han presentado un intento suicida previo, demostrando con ello que el mientras que $11.80 \%$ (18) muestran un riesgo se encuentra presente (figura 3 ).

\section{Gráfica 3. Evaluación del riesgo suicida}

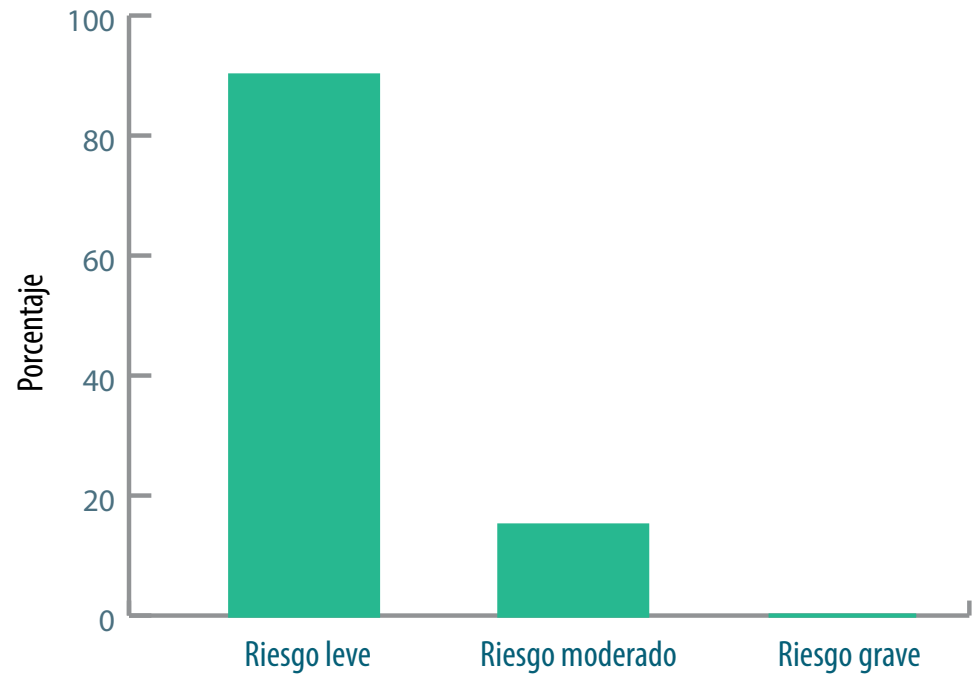

Fuente: Instrumento aplicado a adolescentes de una secundaria general en Aguascalientes. Septiembre de $2019(n=154)$

Niveles de riesgo suicida 
En la pregunta "¿Has pensado alguna vez terminar con tu vida?" del cuestionario para la detección de riesgo suicida en adolescentes, el riesgo suicida está presente en el $45.5 \%$ de los estudiantes evaluados, de los cuales el $33.8 \%$ respondieron "algunas veces"; correspondiendo esto a un riesgo moderado y $11.7 \%$ respondieron que muchas veces han tenido este pensamiento, perteneciendo esto a un riesgo grave (figura 4).

\section{Gráfica 4. ¿Has pensado alguna vez terminar con tu vida?}

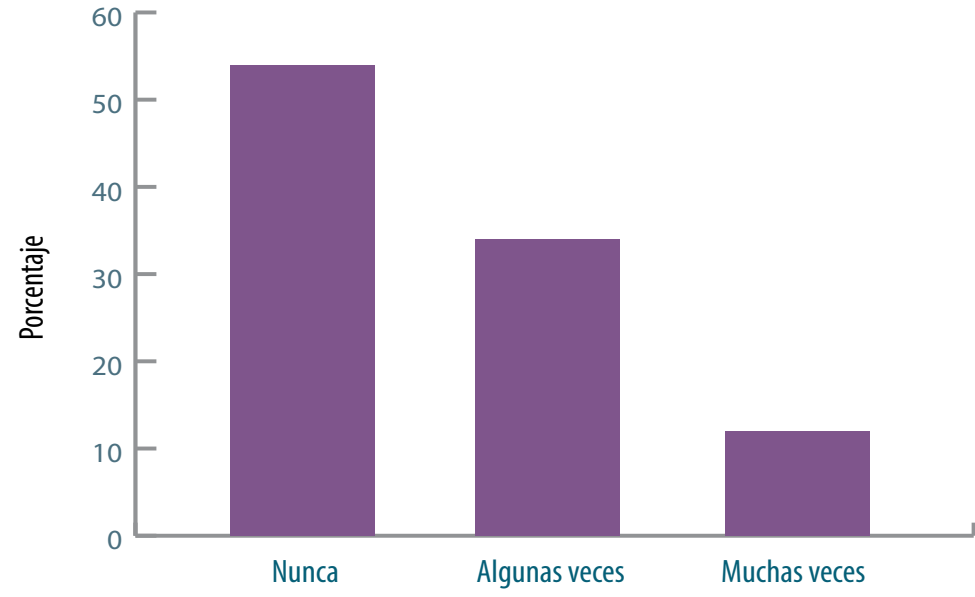

Fuente: Instrumento aplicado a adolescentes de una secundaria general en Aguascalientes. Septiembre de $2019(n=154)$
En la pregunta "¿Has intentado suicidarte?" del cuestionario para la detección del riesgo suicida en adolescentes, arrojó que el $25.3 \%$ (39) manifestó que algunas veces había intentado suicidarse, mientras que el $4.5 \%$ (7) respondió que muchas veces había intentado suicidarse, acudien- do a este acto en forma de solución a sus problemas. Este resultado se considera que es el más relevante del estudio, ya que 46 adolescentes han afirmado haber intentado suicidarse, esto es alarmante ya que el principal predictor del suicidio es el intento previo (figura 5).

\section{Gráfica 5. ¿Has intentado suicidarte?}

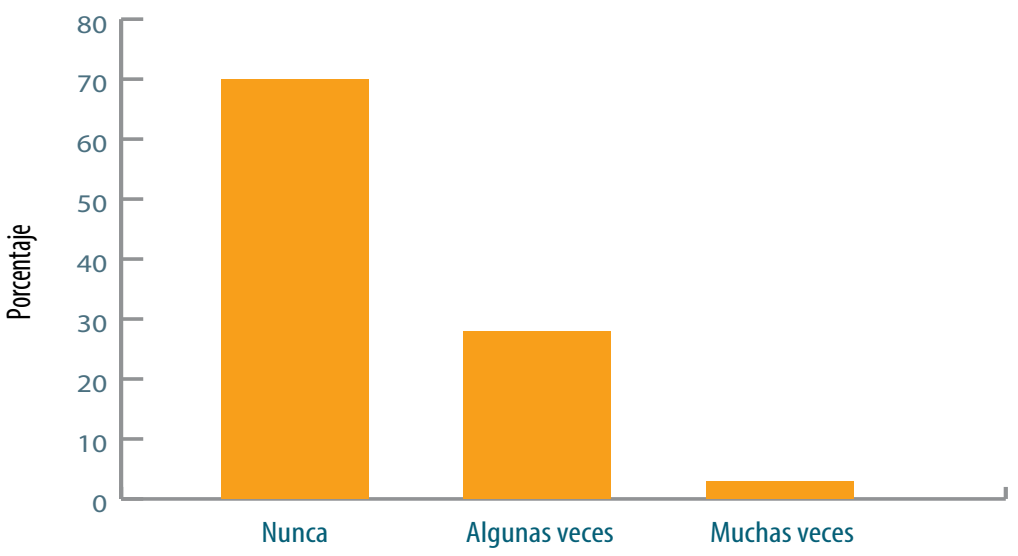

Fuente: Instrumento aplicado a adolescentes de una secundaria general en Aguascalientes. Septiembre de 2019 ( $n=154)$ 
La correlación entre el riesgo suicida y el funcionamiento familiar obtuvo un valor de significancia de $p=.001$, dado esto se aceptó la $\mathrm{Hi}$, confirmando así que el funcionamiento familiar sí tuvo relación con el riesgo suicida en adolescentes de una se- cundaria general de Aguascalientes. $Y$ un coeficiente de correlación de Rho Spearman -.258, mostrando una relación inversa baja, manifestándose como a mayor funcionamiento familiar, menor riesgo suicida (tabla 1).

\section{Tabla I}

\section{Correlación entre el funcionamiento familiar y el riesgo suicida}

\begin{tabular}{|l|c|c|c|}
\hline Variables de correlación & $\begin{array}{c}\text { Coeficiente de corre- } \\
\text { lación }\end{array}$ & Sig. (bilateral) \\
\hline & Rho & $\mathrm{N}$ \\
\hline $\begin{array}{l}\text { Funcionamiento familiar y } \\
\text { riesgo suicida }\end{array}$ & -.258 & .001 & 154 \\
\hline
\end{tabular}

Fuente: Instrumento aplicado a adolescentes de una secundaria general en Aguascalientes. Septiembre de 2019 (n=154). Nota: Rho= Coeficiente de correlación de Rho Spearman; $p=$ Significancia estadística

En la muestra, la edad que presentó mayor riesgo suicida correspondió a 13 años con $35.7 \%$, y esto ocurrió debido a que la mayoría de los adolescentes encuestados tenían esa edad, siendo el riesgo leve el predominante con $31.8 \%$. con una significancia de $p=.030 ; y$ con un coeficiente de correlación de $\mathrm{Rho}=.175$ tendiendo a ser muy bajo el nivel de asociación (tabla 2).

\section{Tabla 2}

\section{Asociación entre el riesgo suicida y la edad (en años) del adolescente}

\begin{tabular}{|c|c|c|c|c|c|c|c|c|c|}
\hline & & \multicolumn{8}{|c|}{ EDAD DEL ADOLESCENTE } \\
\hline \multirow{4}{*}{$\begin{array}{l}\text { RIESGO } \\
\text { DE } \\
\text { SUICIDIO }\end{array}$} & & 11 & 12 & 13 & 14 & 15 & 16 & TOTAL & P (Sig.) \\
\hline & Riesgo leve & $12.3 \%$ & $28.6 \%$ & $31.8 \%$ & $14.3 \%$ & $1.3 \%$ & $0 \%$ & $88.3 \%$ & \multirow{4}{*}{.030} \\
\hline & $\begin{array}{c}\text { Riesgo } \\
\text { moderado }\end{array}$ & $0 \%$ & $3.2 \%$ & $3.9 \%$ & $4.5 \%$ & $0 \%$ & $0 \%$ & $11.7 \%$ & \\
\hline & Riesgo grave & $0 \%$ & $0 \%$ & $0 \%$ & $0 \%$ & $0 \%$ & $0 \%$ & $0 \%$ & \\
\hline \multicolumn{2}{|c|}{ TOTAL } & $12.3 \%$ & $31.8 \%$ & $35.7 \%$ & $18.8 \%$ & $1.3 \%$ & $0 \%$ & $100 \%$ & \\
\hline
\end{tabular}

Fuente: Instrumento aplicado a adolescentes de una secundaria general en Aguascalientes. Septiembre de $2019(n=154)$.

Nota: $p=$ significancia estadística 
Por último, en la muestra, la edad que presentó mayor funcionamiento familiar correspondió a 13 años con 35\%. Siendo el funcionamiento familiar moderadamen- te funcional el predominante con $26 \%$. Con una significancia de $p=.047$ y con un coeficiente de correlación de Rho $=-.161$ (tabla 3).

\section{Tabla 3}

\section{Asociación entre funcionamiento familiar y edad (en años) del adolescente}

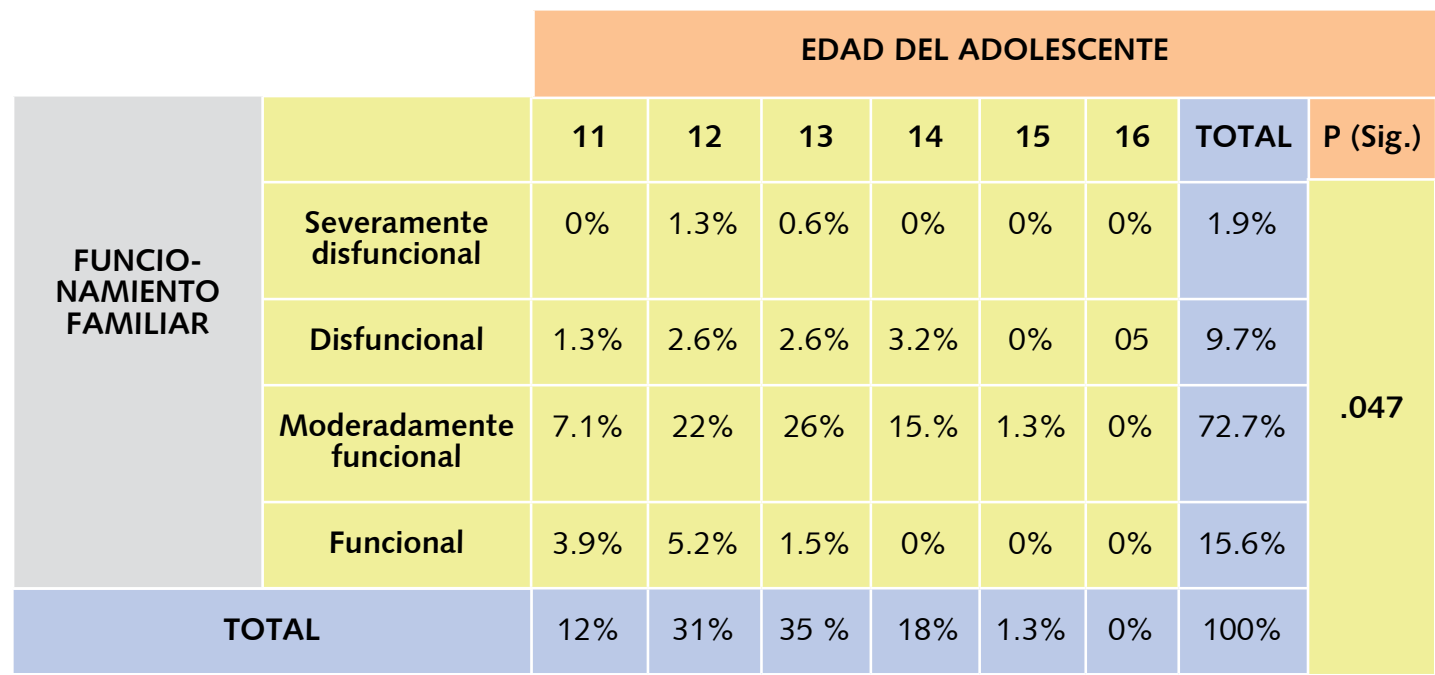

Fuente: Instrumento aplicado a adolescentes de la Secundaria General No. 10 "22 de octubre". Septiembre de 2019.

Nota: $p=$ significancia estadística

\section{Discusión}

Los resultados obtenidos en la presente investigación coinciden con otras investigaciones que se han realizado donde se manifiestan dificultades en el funcionamiento familiar sobre todo en la incapacidad para establecer comunicaciones claras y directas entre sí.

Como lo vemos en la investigación de Pérez, Téllez, Vélez \& Ibáñez ${ }^{13}$ donde estudiaron los factores asociados con los comportamientos suicidas, refieren que existe el riesgo de comportamiento suicida entre mayor sea la disfunción familiar.

Así mismo se coincide con Rodríguez Calle y cols. ya que se tiene una similitud en los resultados en donde ellos afirman tener una correlación significativa con el funcio- namiento familiar y el riesgo suicida ${ }^{14}$ así como en la presente investigación, donde se obtiene que fue baja e inversa, lo que indica que, a mayor funcionamiento familiar, menor es el riesgo suicida en los adolescentes.

El funcionamiento familiar muestra una tendencia a lo moderadamente funcional, lo que indica que los adolescentes no logran un acercamiento positivo y efectivo con el núcleo familiar, al igual que una comunicación asertiva.

Los resultados que se obtienen en cuanto a la evaluación de nivel de riesgo suicida demuestran que de los 154 adolescentes evaluados, predomina un riesgo leve 136 $(88.2 \%)$, seguido de riesgo moderado 18 $(11.8 \%)$. Esto indica que dentro de los 
aspectos que el instrumento evalúa, los adolescentes presentan algún tipo de idea suicida, provocando así que los resultados demuestren este tipo de riesgo, que, aunque este no llega a ser grave, se encuentra latente.

Se coincide con Eguiluz Romo y cols. ${ }^{8}$ cuando afirman que los adolescentes que tienen un funcionamiento familiar disfuncional pueden llegar a presentar un riesgo suicida entre leve y moderado, siendo el $1.9 \%$ de los estudiantes encuestados los que presentan un funcionamiento familiar severo disfuncional, esto se refleja por la falta de comunicación que implica que entre más clara y directa sea, más eficiente será el funcionamiento familiar.

También los resultados que se obtienen arrojan datos importantes en cuanto a los pensamientos de terminar con su vida con un porcentaje de $34 \%$ algunas veces y un $11.8 \%$ refiere haber tenido muchas veces estos pensamientos, con esto se coincide de igual manera con la investigación de Leal y Vázquez (2012), donde los adolescentes estudiados manifiestan que algunas veces en sus vidas experimentaron deseos de terminar con su vida (38.5\%). ${ }^{15}$

En la investigación de Guibert y Torres, refiere que existen factores familiares predisponentes para que exista una conducta suicida, los cuales son riñas constantes, abandono emocional o físico, hostilidad e incomunicación, desesperanza, rechazo familiar, inconsistencia y desorganización, falta de comunicación, hostilidad, coincidiendo así con algunos de los factores del funcionamiento familiar disfuncional.

Se difiere con ellos en su investigación Intento suicida y funcionamiento familiar, donde se aplica una prueba de funcionamiento familiar y muestra predominantemente el funcionamiento funcional con un $58,1 \%$ y en la presente investigación el nivel predominante es moderadamente funcional con un $72.7 \% .^{16}$

Así mismo, los resultados encontrados por Sánchez Villarreal y colaboradores ${ }^{17}$ en su investigación La ideación suicida en adolescentes: un análisis psicosocial coinciden con los de la presente investigación ya que muestran que hay una relación con el funcionamiento familiar y el riesgo suicida, añadiendo que la adecuada integración familiar puede ser considerada como un factor protector contra la ideación del suicidio.

En la investigación Funcionamiento familiar e ideación suicida en alumnos de 5to año de educación secundaria del distrito de San Juan de Miraflores de Pérez Carrillo, se encuentra que no existe relación entre el funcionamiento familiar y el riesgo de ideación suicida en adolescentes masculinos, pero sí existe una relación $(p=0.003)$ en las adolescentes femeninas. Se difiere con ellos, ya que ellos basan sus criterios de correlación tomando en cuenta el sexo de los adolescentes y la presente investigación toma en cuenta la edad en relación con el funcionamiento familiar. ${ }^{18}$

La relación que se hizo de acuerdo con la edad y el funcionamiento familiar muestra que los adolescentes de 13 años de edad presentan mayor funcionamiento familiar, sin embargo en la investigación de Burgos y colaboradores ${ }^{19}$ en donde se habla sobre el análisis de los componentes funcionales de una dinámica familiar y los factores asociados al riesgo de intento de suicidio se obtiene una correlación positiva débil entre las variables del funcionamiento familiar y la edad, donde se afirma en su investigación que, a mayor edad, la percepción de funcionamiento familiar mejoraría levemente, al contrario de la presente investigación donde los adolescentes de mayores edades no muestran una mejoría de esta percepción de funcionabilidad con la familia.

En la investigación de Paez, Santander y cols. ${ }^{20}$ manejan el término de adaptabilidad familiar como un funcionamiento familiar aceptable y en donde no existe una correlación con la adaptabilidad y el riesgo de intento suicida ya que las variables son 
estudiadas de manera individual y se toman en cuenta otros factores para la relación y aceptación de las variables, como lo son el hecho de que en la familia ya se había presentado un intento previo de suicidio. Por lo tanto y por la naturaleza de esta investigación, se encontraron resultados diferentes, pero no necesariamente una diferencia de opinión, dado que los estudios no son directamente comparables entre sí.

Si bien en la presente investigación se estudia además la relación de la edad de los adolescentes con el riesgo suicida, obteniendo así que la edad que muestra un riesgo de suicidio leve es de 13 años, tal como se observa en la investigación de Hernández-Trujillo ${ }^{21}$ y cols. puesto que en su investigación la frecuencia de edades de presentar riesgo de intento suicida fue entre los 10 y 19 años. Se coincide con ellos ya que se maneja un rango de edad donde incluye la de los adolescentes participantes de esta investigación.

\section{Conclusiones}

Se concluye que el funcionamiento familiar predominante entre los adolescentes es moderadamente funcional, siendo el factor de comunicación el más alterado, debido a que la mayoría presenta una comunicación disfuncional con sus familiares; mientras que para el riesgo suicida se demuestra que gran parte de los adolescentes presentan un riesgo leve. Sin embargo, algunos de ellos refieren haber pensado una vez el terminar con su vida y otros más han manifestado realizar de uno a varios intentos de suicidio. Por esta razón es importante que se tomen medidas de prevención ya sea con padres o tutores de los alumnos, así como también capacitación continua al personal docente, para que puedan identificar en los adolescentes datos de riesgo, y así poder canalizar la atención a psicología para su correcto tratamiento y evitar que los adolescentes atenten contra su vida.

Por lo anterior se tienen los suficientes elementos para afirmar que sí existe una relación inversa baja entre el funcionamiento familiar y el riesgo suicida en los adolescentes de esta secundaria.

\section{Bibliografía}

1. Suicidio [Internet]. Organización Mundial de la Salud 2018 [citado 17 de noviembre de 2018]. Disponible en: http://www.who.int/topics/suicide/es/

2. INEGI. Estadísticas a propósito del día mundial para la prevención del suicidio datos nacionales [Internet]. 2020 [citado el 15 de febrero de 2021]. Disponible en: https:// www.inegi.org.mx/contenidos/saladeprensa/aproposito/2020/suicidios2020_Nal.pdf

3. Estadísticas a propósito del... día mundial para la prevención del suicidio (10 de septiembre)" [Internet]. INEGl. 2018 [citado 17 de noviembre 2018]. Disponible en: http://www.inegi.org.mx/saladeprensa/aproposito/2016/suicidio2016_0.pdf

4. El suicidio en infancia y adolescencia [Internet]. Guiasalud. es. 2018 [citado el 17 de noviembre 2018]. Disponible en http://www.guiasalud.es/egpc/depresion_infancia/completa/documentos/apartado09/apartado09.pdf

5. Organización Mundial de la Salud. (2018). Desarrollo en la adolescencia. [Internet] [Citado 26 de septiembre 2018]. Disponible en: http://www.who.int/maternal_child_adolescent/topics/adolescence/dev/es/

6. Arellano López J, Velasco Campos M. Instrumentos de evaluación en terapia familiar y de pareja. 1st ed. México, D.F.: Editorial Pax; 2006.

7. Cartilla Educativa Para La Prevención Del Suicidio En Adolescentes [Internet]. calameo.com. 2019 [citado 25 noviembre 2019]. Disponible en: https://es.calameo.com/ $\mathrm{read} / 004959318 \mathrm{dd} 7 \mathrm{e} 63 \mathrm{bafc} 80$

8. Eguiluz Romo, Luz de Lourdes, Ayala Mira, Mónica, Relación entre ideación suicida, depresión y funcionamiento familiar en adolescentes. Psicología Iberoamericana [Internet]. 2014:22(2):72-80. Recuperado de: https://www. 
redalyc.org/articulo.oa?id=133938134009

9. OMS. Organización Mundial de la Salud. [Internet]. [Citado 17 de febrero 2018]. Disponible en: http://www.who. int/topics/depression/es/

10. Estadísticas a propósito del... día mundial para la prevención del suicidio [Internet]. INEGI. 2017 [citado 17 de febrero 2018]. Disponible en: http://www.inegi.org.mx/ saladeprensa/aproposito/2017/suicidios2017_Nal.pdf

11. Ley General de Salud [Internet]. 1st ed. México D.F: DOF; 2018 [citado 17 de Febrero de 2019]. Disponible en: http://www.salud.gob.mx/unidades/cdi/nom/compi/ rlgsmis.html

12. Declaración del Helsinki [Internet]. 1st ed. Declaración de Helsinki; 2018 [citado 17 de febrero de 2019]. Disponible en: http://www.conamed.gob.mx/prof_salud/pdf/helsi

13. Pérez, I., Téllez, A., Vélez, L., Ibáñez, M. (2012). Factores asociados con comportamiento suicida en adolescentes estudiantes de octavo grado en tres colegios bogotanos (Tesis de licenciatura). Recuperado de http://www.redalyc. org/pdf/806/80624093004.pdf

14. Martha Elizabeth Rodríguez Calle, Pedro Pablo Huanca Casa. Funcionamiento familiar e ideación suicida en estudiantes del nivel secundario de una institución educativa de Puno. Universidad Peruana Unión (Tesis de licenciatura) [internet] 2019;10 (1). Recuperado de: https://repositorio.upeu.edu.pe/handle/UPEU/2833

15. Leal Zavala, Rafael A. Vásquez Rodríguez, Luis E. Ideación suicida en adolescentes cajamarquinos prevalencia y factores asociados. Universidad Privada Antonio Guillermo Urrelo Facultad de Psicología. [internet] Dic 2012. Disponible en: http://bp000695.ferozo.com/wp-content/ uploads/2013/01/Ideacion-suicida-en-adolescentes-cajamarquinos.pdf

16. Guibert Reyes Wilfredo, Torres Miranda Niurka. Intento suicida y funcionamiento familiar. Rev. Cubana Mes Gen Integr [Internet]. 2001 Oct [citado 2020 Mayo 10]; 17(5):452-460. Disponible en: http:// scielo.sld.cu/scielo.php?script $=$ sci_arttext\&pid $=$ S0864$21252001000500008 \& \operatorname{lng}=$ es
17. Sánchez-Sosa Juan Carlos, Villarreal-González María Elena, Musitu Gonzalo, Martínez Ferrer Belén. Ideación suicida en adolescentes: un análisis psicosocial. Psychosocial Intervention [Internet]. 2010 Dic [citado 2020 mayo 12]; 19(3): 279-287. Disponible en: http:// scielo.isciii.es/scielo.php?script=sci_arttext\&pid $=$ S1132$05592010000300008 \& \operatorname{lng}=\mathrm{es}$.

18. Pérez-Carrillo P. Funcionamiento familiar e ideación suicida en alumnos de 5 to año de educación secundaria del distrito de San Juan de Miraflores. Universidad César Vallejo Lima norte [internet] Psique Mag, 2016(4)(1). Recuperado de: http://webcache.googleusercontent.com/search?q= cache:J9kUfgWfJ4cJ:revistas.ucv.edu.pe/index.php/psiquemag/issue/download/210/Psiquemag\% 25202015$15+\& c d=9 \& h l=e s \& c t=c \operatorname{lnk} \& g l=m x$

19. Burgos Graciela, Narváez Nilda, Mercedes-Bustamante Pamela, Burrone María, Fernández Ruth, Abeldaño Roberto. Funcionamiento familiar e intentos de suicidio en un hospital público de Argentina. Acta de Investigación Psicológica [internet] Dic 2017 [citado mayo 2020]; 17 (7). Disponible en: http://www.scielo.org.mx/pdf/aip/v7n3/2007-4719aip-7-03-2802.pdf

20. Pavez Paula, Santander Natalia, Carranza Jorge, VeraVillarroel Pablo. Factores de riesgo familiares asociados a la conducta suicida en adolescentes con trastorno depresivo. Rev. méd. Chile [Internet]. 2009 Feb [citado 2020 Mayo 12]; 137(2): 226-233. Disponible en: $\quad$ https://scielo.conicyt.cl/scielo.php?script=sci_ arttext\&pid =S0034-98872009000200006\&lng $=$ es. http://dx.doi.org/10.4067/S0034-98872009000200006

21. Hernández Trujillo Ariane, Rebustillo Escudero Gloria Teresita, Danauy Enamorado Marlene de la C, Bess Constantén Sonia. Influencia del medio familiar en un grupo de 5 a 19 años con riesgo suicida. Rev Cubana Med Gen Integr [Internet]. 1999 Ago [citado 2020 mayo 12]; 15(4): 372-377. Disponible en: http://scielo.sld.cu/scielo.php?script $=$ sci_ arttext\&pid $=$ S0864-21251999000400005\&lng $=$ es 